\title{
Diabetes mellitus tipo 3c secundaria a pancreatitis crónica: reporte de un caso
}

Cintya Marisol Guibar Deza*1, a; José Guillermo Cabanillas Lopez², b; Evelyn del Socorro Goicochea Ríos , c

RESUMEN

La diabetes mellitus secundaria a enfermedades pancreáticas está clasificada como diabetes pancreatogénica o tipo $3 c$ (DM3c), y es clínica y metabólicamente distinta de las otras formas de diabetes. Se presenta el caso de una paciente de 35 años de edad con historia de dolor abdominal superior episódico crónico, que consume alcohol desde los 17 años y ha sido diagnosticada de diabetes mellitus tipo 2. Consulta por una infección urinaria complicada y por dolor agudo en el abdomen superior. Durante la hospitalización presentó episodios de hiperglicemia seguidos de hipoglicemias, con insulinoterapia administrada bajo supervisión. Este caso nos motiva para estudiar a los pacientes diabéticos con historia de dolor abdominal mal referido o signos de malabsorción y mejorar la intervención sanitaria para prevenir o tratar la desnutrición, controlar la esteatorrea y minimizar la hiperglucemia inducida por las comidas o estilos de vida.

Palabras clave: Pancreatitis crónica; Diabetes mellitus; Páncreas (Fuente: DeCS BIREME).

\section{Type $3 c$ diabetes mellitus secondary to chronic pancreatitis: a case report}

\begin{abstract}
Diabetes mellitus secondary to pancreatic diseases is classified as pancreatogenic diabetes or type $3 c$ diabetes mellitus (T3CDM), which is clinically and metabolically different from the other types of diabetes. We present the case of a 35-year-old female patient with a history of chronic episodic upper abdominal pain, alcohol consumption since she was 17, and type 2 diabetes mellitus. She sought medical attention because of a complicated urinary tract infection in addition to acute upper abdominal pain. During hospitalization, she presented episodes of hyperglycemia followed by hypoglycemia with insulin therapy administered under supervision. This case encourages us to delve into the study of diabetic patients with a history of poorly referred abdominal pain or malabsorption signs, thus improving health intervention to prevent or treat malnutrition, control steatorrhea, and reduce hyperglycemia induced by meals or lifestyles.
\end{abstract}

Keywords: Pancreatitis, chronic; Diabetes mellitus; Pancreas (Source: MeSH NLM).

1 Universidad César Vallejo, Facultad de Ciencias Médicas. Trujillo, Perú.

2 Universidad Nacional de Trujillo. Trujillo, Perú.

a Médico Residente Medicina Familiar y Comunitaria.

b Médico Residente Medicina Interna.

c Docente segunda especialización.

*Autor corresponsal. 


\section{INTRODUCCIÓN}

La pancreatitis crónica (PC) es una enfermedad poco común que implica inflamación, fibrosis y pérdida de células acinares e islotes de forma progresiva e irreversible ${ }^{(1-3)}$; además, es un problema de salud importante que puede manifestarse con dolor abdominal implacable, complicaciones peripancreáticas, insuficiencia pancreática exocrina (EPI) y endocrina, desnutrición a largo plazo y diabetes mellitus tipo $3 c^{(1,2)}$.

La prevalencia anual reportada por cada 100000 personas es de 26 y 42 casos en Europa y Estados Unidos, respectivamente. La incidencia global en las últimas tres décadas es de, aproximadamente, 4/100000 personas-año. En Latinoamérica, la incidencia y prevalencia de la PC no es bien conocida, pero se presume que su frecuencia se ha incrementado en las últimas décadas ${ }^{(3)}$.

Las principales características clínicas de la pancreatitis son dolor abdominal localizado en el abdomen superior a medio, distensión abdominal, náuseas, fiebre, dolor en el costado, vómitos, dolor de espalda, ictericia, hematemesis, diarrea, melena con mal olor, deposiciones grasas y pérdida de peso ${ }^{(4)}$.

En la PC, el reemplazo progresivo del parénquima pancreático con fibrosis puede provocar daños irreversibles en los islotes pancreáticos y riesgo de diabetes mellitus. Esta forma de diabetes pancreática, también llamada diabetes mellitus tipo 3c (DM3c), puede ocurrir en más de la mitad de los pacientes con pancreatitis crónica grave de larga evolución, y se caracteriza clínicamente por la pérdida de la masa de los islotes ${ }^{(5)}$.

La DM3c suele ser etiquetada como diabetes tipo 2 (87,8 \%), pero tiene un curso clínico diferente con mal control glucémico y un requerimiento marcadamente mayor de insulina. La incidencia de aparición de DM3c es 2,59/100 000 personas-año, y la edad promedio de aparición es 59 años. Entre los factores de riesgo se señalan el alcoholismo, el tabaquismo, el sobrepeso u obesidad y la raza blanca ${ }^{(6)}$.

\section{CASO CLÍNICO}

Paciente mujer de 35 años de edad con diagnóstico de diabetes mellitus tipo 2 hace dos años. Tiene un tratamiento irregular con glibenclamida y diagnóstico de hipotiroidismo subclínico hace un mes que se trata con levotiroxina 75 $\mathrm{mcg} /$ día. Ingresa al servicio de emergencia por dolor abdominal y vómitos, con un tiempo de enfermedad de aproximadamente 15 días. Durante este tiempo presentó epigastralgia tipo cólico asociada a náuseas y vómitos postprandiales de contenido alimentario que se repite a los cuatro y a los diez días después del primer episodio y que cede con medicamentos que no refiere; posteriormente, se añade cefalea y tos no productiva. Además, reporta pérdida de peso (10 kg en dos meses). El día del ingreso vuelve a presentar la sintomatología y dificultad respiratoria, por lo que decide acudir a emergencia. Como antecedente figura el consumo de alcohol desde los 17 años, aproximadamente una vez por semana, que es ingerido hasta embriagarse. Tiene cuatro hijos, todos los que fueron macrosómicos al nacer. Durante su segundo embarazo refiere un diagnóstico de "enfermedad del páncreas" (no recuerda el nombre), motivo por el que fue hospitalizada, y también el diagnóstico de diabetes gestacional durante su último embarazo. Refiere una historia de dolor abdominal superior episódico crónico a partir de su segundo embarazo que podía tolerar si usaba analgésicos, por lo cual nunca consultó a un médico por esta molestia. Su madre y abuela materna tienen diagnóstico diabetes mellitus 2.

En el examen presenta un regular estado general. Ventila espontáneamente, sin dificultad. Tiene una $\mathrm{SatO}_{2}: 95 \%$, PA: $80 / 60 \mathrm{mmHg}$ y las demás funciones vitales eran estables. Peso $=44 \mathrm{~kg}$, talla $=1,58 \mathrm{~m}, I M C=17,6 \mathrm{~kg} /$ $\mathrm{m}^{2}$, pálida $(+/+++)$, moderado pasaje del murmullo vesicular sin ruidos agregados y dolor a la palpación profunda en epigastrio e hipocondrio derecho. En el resto de la evaluación no muestra nada particular. Se solicitan exámenes auxiliares y se inicia tratamiento con escopolamina e hidratación con $\mathrm{NaCl} 0,9 \%$. Exámenes auxiliares: glicemia $=670 \mathrm{mg} / \mathrm{dL}$; creatinina $=0,94 \mathrm{mg} /$ $\mathrm{dL}$; urea $=24 \mathrm{mg} / \mathrm{dL}$; hemograma $=13320$ leucocitos $/$ uL (Ab:4 \%, Seg:73\%, Eo:2 \%, Mon:1 \%, Linf:20 \%); Hb= $10,3 \mathrm{~g} / \mathrm{dL} ; \mathrm{Hto}=32,3 \%$; plaquetas $=684000 / \mathrm{uL}$; examen de orina (+); Gram s/c= bacilos gramnegativos, 90-95 leucocitos/campo, 8-10 hematíes/campo, trazas de glucosa, nitritos $(++/+++)$. AGA y electrolitos: $\mathrm{pH}=7,348$; $\mathrm{PCO}_{2}=39,3 \mathrm{mmHg} ; \mathrm{HCO}_{3}=21,8 \mathrm{mmol} / \mathrm{L} ; \mathrm{Na}+=123,1$ $\mathrm{mmol} / \mathrm{L} ; \mathrm{K}+=3,26 \mathrm{mmol} / \mathrm{L} ; \mathrm{Cl}-=89,2 \mathrm{mmol} / \mathrm{L} ; \mathrm{Ca}++=$ $0,65 \mathrm{mmol} / \mathrm{L} ; \mathrm{Mg}++=0,23 \mathrm{mmol} / \mathrm{L}$. Inician el tratamiento con insulina R $10 \mathrm{Ul}$ stat y luego a escala. También solicitan un urocultivo e inician la antibioticoterapia. Se indica realizar controles glicémicos en horario y la paciente se hospitaliza. La antibioticoterapia continúa hasta que en el urocultivo aparece una bacteria nosocomial y varía la sensibilidad antibiótica, entonces el tratamiento cambia a imipenen y la evolución continúa de manera favorable. Se inicia terapia con insulina NPH $22 \mathrm{UI} /$ día, la cual se aumenta hasta un máximo de $52 \mathrm{UI} /$ día en un periodo de 15 días; sin embargo, no se logró un adecuado control y la paciente continúa con picos de hiperglicemia y, en algunas oportunidades, seguidos de hipoglicemias de hasta $36 \mathrm{mg} /$ $\mathrm{dL}$, por lo que se decide cambiar la insulina NPH por insulina glargina, la cual se inicia con $10 \mathrm{UI} /$ día y se aumenta hasta $32 \mathrm{UI} / d i ́ a$. Después, se añade insulina tipo aspart $5 \mathrm{UI}$ con el almuerzo y $5 \mathrm{UI}$ con la cena, con lo que se logra un mejor control. Se le solicitó una ecografía (Figura 1), la que muestra litiasis múltiple en el conducto de Wirsung que está dilatado, por lo que se solicita una colangioresonancia 
que confirma la litiasis en el conducto de Wirsung y su dilatación, así como atrofia pancreática difusa severa, con lo que se diagnostica la pancreatitis crónica. La paciente es dada de alta luego de 30 días de hospitalización con la remisión del cuadro infeccioso, un mejor control glicémico con insulina glargina $26 \mathrm{UI} /$ día, insulina tipo aspart $5 \mathrm{UI}$ con el almuerzo y $5 \mathrm{UI}$ con la cena, y el tratamiento enzimático, así como con indicaciones de control por gastroenterología.

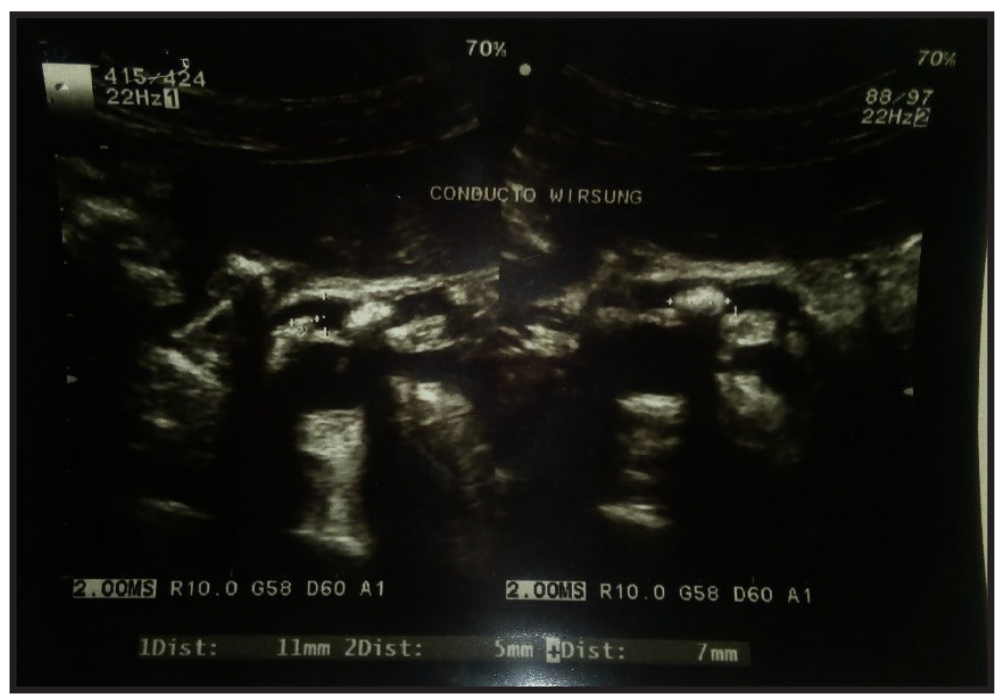

Figura 1. Estudio ecográfico que evidencia el conducto de Wirsung dilatado $(7 \mathrm{~mm})$, en cuyo interior se observan múltiples imágenes litiásicas, la mayor de $11 \mathrm{~mm}$

\section{DISCUSIÓN}

La diabetes mellitus secundaria a enfermedades pancreáticas es clasificada como diabetes pancreatogénica o diabetes

mellitus tipo 3c (DM3c) y es la causa más comúnmente identificada la pancreatitis crónica (Figura 2).

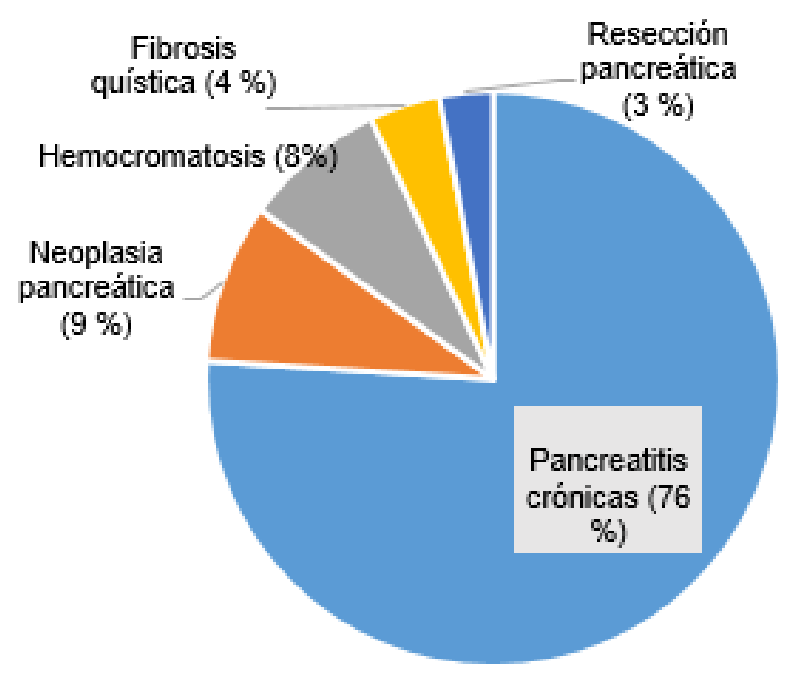

Figura 2. Distribución de las causas de diabetes mellitus tipo 3c. Adaptado de Andersen D et al. Diabetes, Pancreatogenic Diabetes, and Pancreatic Cancer (15) 
El diagnóstico de $\mathrm{DM} 3 \mathrm{c}$ en un paciente con pancreatitis crónica conocida puede no ser tan difícil, y todo paciente con pancreatitis crónica debe ser monitoreado por el desarrollo de DM3c al menos una vez al año ${ }^{(7)}$. Sin embargo, la clasificación correcta a menudo se pasa por alto y se reporta que hasta $78,3 \%$ de los pacientes se clasifican como diabetes tipo $2^{\left({ }^{(6)}\right.}$. En nuestro caso, la paciente había sido diagnosticada con DM2 hace dos años, y a pesar del tiempo transcurrido, su requerimiento de insulina fue bastante elevado.

Reconocer el papel del daño pancreático en el desarrollo de diabetes del paciente es vital para un adecuado plan de manejo; si esta alteración no se identifica, la fisiología que se observa puede resultar en un tratamiento subóptimo o errado ${ }^{(6)}$. A diferencia de los otros tipos de diabetes, en la DM3c la inflamación o pérdida de islotes involucra a las células $B$ y también a las células del polipéptido pancreático (PP) al inicio de la enfermedad, y a las células A al final del cuadro. Por ello, la producción de glucagón disminuye, lo que podría explicar los episodios de hipoglicemia severa. Debido a la insuficiencia exocrina pancreática concomitante, también existe una mala digestión de nutrientes con el consiguiente deterioro en la secreción de incretina ${ }^{(8)}$. La complicación adicional de la mala absorción de nutrientes y la dieta oral, con frecuencia deficiente (para evitar síntomas), hace que el paciente con DM3c tenga un alto riesgo de desnutrición e hipoglucemia crítica por lo que, adicionalmente, se requiere terapia de reemplazo enzimático y vitamina $D$ para prevenir malnutrición y osteoporosis ${ }^{(6-16)}$. En el presente caso la paciente tiene un IMC de $17,6 \mathrm{~kg} / \mathrm{m}^{2}$, lo cual sugiere un estado de malnutrición.

Se sabe que los niveles circulantes de insulina son bajos en $\mathrm{DM} 3 \mathrm{C}$. Junto con un aumento compensatorio en la sensibilidad periférica a la insulina, hay una disminución en la sensibilidad hepática a la insulina y la no supresión de la producción de glucosa hepática, lo que impulsa la hiperglucemia. Dichos cambios están asociados con la reducción de la secreción pancreática de PP (la cual aumenta la expresión de receptores de insulina en el hígado), por lo que su pérdida puede llevar a una resistencia hepática a la insulina, una diferencia fisiológica importante con la DM1 ${ }^{(6)}$. El origen de esta alteración no es la resistencia a la insulina, sino el descenso de su producción debido a la reducción de la cantidad de células productoras. Esto conlleva a una disminución neta de insulina que puede derivar en alteración del índice de HOMA (del inglés, homeostatic model assessment), sin alteración inicial de la sensibilidad de los receptores. Posteriormente, de forma compensatoria, aumenta la sensibilidad a la insulina a medida que van disminuyendo las unidades productoras. Finalmente, la disminución de la producción de insulina llega a puntos críticos y se hace necesario el tratamiento sustitutorio. Es entonces cuando el factor "sensibilidad" juega un rol importante, debido a la variable respuesta al tratamiento. Por lo tanto, la DM asociada con la enfermedad pancreática es de naturaleza errática, caracterizada por cambios significativos en la glucosa en sangre de hipoglucemia a hiperglucemia de una manera que es difícil de controlar, a pesar de mínimos cambios en las dosis terapéuticas ${ }^{(9)}$. En el presente caso las glicemias de la paciente fueron de difícil manejo, ya que con la misma dosis de insulina +/- $1 \mathrm{Ul}$ administrada por personal de enfermería durante la hospitalización, variaba de hiperglicemia a hipoglicemia de un control para el siguiente.

La incidencia global de pancreatitis aguda es 34 por cada 100000 personas al año en la población general; para pancreatitis crónica es 10 casos, y para diabetes mellitus postpancreatitis es de seis casos. La tasa de transición global desde el primer episodio de pancreatitis aguda a un episodio recurrente es de $20 \%$ y de pancreatitis aguda recurrente a la pancreatitis crónica es de $35 \%$. En este caso, el episodio de "enfermedad del páncreas" durante el segundo embarazo fue, probablemente, un cuadro de pancreatitis aguda (aunque no está bien documentado, ya que la historia clínica no se encontró). La pancreatitis aguda (incluida su forma no necrosante) conduce a una serie de secuelas mucho después de la resolución clínica $y$, por lo tanto, ya no debe considerarse una enfermedad autolimitada. Una de estas secuelas es la diabetes mellitus postpancreatitis (la más frecuente), que está causada por la pancreatitis aguda y aguda recurrente en el $80 \%$ de los casos y por la pancreatitis crónica en el $20 \%{ }^{(10)}$.

A menudo, en la $\mathrm{PC}$, los pacientes son sintomáticos durante años antes de poder llegar al diagnóstico, en ellos, el dolor es el síntoma predominante y más incapacitante. Las últimas guías recomiendan un enfoque de tratamiento progresivo que implica un tratamiento médico inicial del dolor seguido de intervenciones que incluyen endoscopia y cirugía. El tiempo promedio desde el inicio de los síntomas hasta el diagnóstico de pancreatitis crónica es de 62 meses, y en personas sin historia de alcoholismo, el tiempo promedio es de 81 meses. La PC es heterogénea en muchos aspectos, como su etiología, estadiaje, duración y pretratamientos; por lo tanto, cada paciente puede requerir un enfoque de tratamiento diferente ${ }^{(11,12)}$. En el presente caso no podemos decir con exactitud cuánto fue el tiempo que tomó desde el episodio de pancreatitis aguda hasta el establecimiento de la pancreatitis crónica; sin embargo, lo que se concluye, según el relato del caso y el padecimiento crónico del dolor abdominal superior episódico, es que hubo una transición de pancreatitis aguda a pancreatitis aguda recurrente $y$, finalmente, a pancreatitis crónica; además, la paciente se automedicó con analgésicos en repetidas oportunidades y no acudió con un especialista, lo cual ocasionó la progresión y diagnóstico tardío de la enfermedad. 
Entre los factores de riesgo para la PC, el alcohol es el que se identifica con mayor frecuencia, y el fumar es dependiente de la dosis para la susceptibilidad y la progresión de la pancreatitis. También se ha encontrado que la acumulación de grasa en el páncreas es un factor de riesgo significativo ${ }^{(13-15)}$. La pancreatitis crónica alcohólica (ACP) y la pancreatitis crónica idiopática (ICP) difieren en muchos aspectos, especialmente en género, edad, complicaciones, morfología del conducto pancreático y tipo de dolor. Los pacientes con ACP, generalmente, tienen una evolución más severa de IPC; y en la ACP la edad de inicio es significativamente menor y el diagnóstico distinto que en los pacientes de ICP ${ }^{(15)}$. Dentro de los hallazgos en estudios de resonancia magnética de pancreatitis crónica avanzada, se evidencia dilatación del conducto pancreático principal, con ectasia de las ramas laterales; otras características incluyen calcificaciones intraductales, las cuales son el hallazgo más específico visto en cerca de la mitad de los pacientes con pancreatitis crónica y atrofia parenquimal. Las calcificaciones intraductales 0 parenquimales son usualmente vistas en la pancreatitis crónica alcohólica pero no en las pancreatitis crónicas debidas a otras causas ${ }^{(16)}$. En el presente caso la paciente refiere un consumo crónico y frecuente de alcohol desde los 17 años; sin embargo, a pesar de que la ACP es más frecuente en varones, el dato de dicho hábito, su temprana edad, los hallazgos compatibles con pancreatitis crónica en estadio avanzado más las imágenes de la ecografía y de la colangioresonancia frecuentemente halladas en ACP orientan a un caso de pancreatitis crónica de etiología alcohólica.

Se ha observado que un 26 a $80 \%$ de pacientes con pancreatitis crónica son diagnosticados con diabetes mellitus, de acuerdo a la cohorte y la duración del seguimiento. La prevalencia de diabetes aumenta con una mayor duración de la enfermedad ${ }^{(17)}$.

La evaluación inicial de pacientes con pancreatitis crónica debe incluir un examen de glucosa en ayunas y HbA1c. Si las pruebas sugieren una tolerancia alterada a la glucosa, se recomienda una evaluación adicional con la prueba

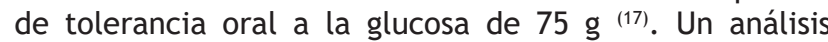
concomitante de los niveles de insulina y/o péptido $C$ puede ser útil para distinguir entre diabetes mellitus tipo 2 y tipo 3c. Una respuesta ausente del polipéptido pancreático (PP) es capaz de distinguir entre la diabetes mellitus tipo $3 c$ del tipo 1 temprano y también puede distinguir el tipo $3 c$ del tipo 2 , que se caracteriza por niveles elevados de polipéptido pancreático ${ }^{(7)}$.

Si bien la DM3c tiene características que se superponen con la DM1 y la DM2, es clínica y metabólicamente distinta de ambas (tablas 1 y 2 ), con características únicas y prioridades de manejo específicas que requieren una terapia personalizada.

Tabla 1. Criterios diagnósticos para DM3c

\section{Criterios diagnósticos}

Mayores (deben estar presentes)

- Insuficiencia pancreática exocrina: Prueba de elastasa-1 fecal monoclonal o pruebas de función directa.

- Ecografía endoscópica, resonancia magnética, tomografía computarizada con imagen patológica del páncreas.

- Marcadores autoinmunitarios asociados con diabetes mellitus tipo 1 ausentes.

Menores

- Secreción de polipéptido pancreático ausente

- Secreción de incretina alterada (p. ej., GLP-1)

- No hay resistencia excesiva a la insulina (p. ej., HOMA-IR)

- Función de las células beta deteriorada (p. ej., HOMA-B, relación péptido-C / glucosa)

- Niveles bajos de vitaminas liposolubles (A, D, E y K)

Adaptado de ${ }^{(7)}$ 
Tabla 2. Características DM1, DM2, DM3C

\begin{tabular}{llll}
\multicolumn{1}{c}{ Parámetro } & DM1 & DM2 & DM3 \\
\hline Cetoacidosis & Común & Rara & Rara \\
\hline Hipoglicemia & Común & Rara & Común \\
\hline Sensibilidad periférica a insulina & Normal o disminuida & Disminuida & Normal o incrementada \\
\hline Sensibilidad hepática a insulina & Normal o disminuida & Disminuida & Normal o disminuida \\
\hline Nivel de insulina & Baja o ausente & Alto o normal & Normal o bajo \\
\hline Nivel de glucagon & Normal o alto & Normal o alto & Normal o bajo \\
\hline Nivel de polipéptido pancreático & Normal o bajo (tardío) & Normal o alto & Bajo o ausente \\
\hline Nivel de polipéptido inhibidor gástrico (GIP) & Normal o bajo & Variable & Bajo \\
\hline Nivel de GLP-1 & Normal & Variable & Variable \\
\hline Edad de inicio típica & Niñez o adolescencia & Adultez & Cualquiera \\
\hline Etiología típica & Autoinmune & Obesidad, edad & PC, fibrosis quística, postquirúrgico \\
\hline Adaptado de ${ }^{(8)}$ & & & \\
\hline
\end{tabular}

El reemplazo oral de la enzima pancreática para corregir la mala digestión, particularmente de grasa, es necesario para optimizar la secreción de incretina y permitir la absorción de vitamina $\mathrm{D}$ y otras vitaminas liposolubles. Si un paciente no se ajusta a la presentación común y se queja de síntomas gastrointestinales, el médico debe considerar la presencia de diabetes mellitus del tipo $3 \mathrm{c}$ e iniciar estudios adicionales ${ }^{(8)}$. En nuestro caso no hubo sospecha de una diabetes DM3c hasta el hallazgo incidental de la enfermedad pancreática en el estudio de imágenes, esto provocó una demora para establecer un manejo adecuado de la paciente. Se consideró como diagnóstico presuntivo de egreso la DM3c, ya que el hospital no contaba con los exámenes auxiliares necesarios para cumplir con los criterios diagnósticos y solo se realizaron los que estaban cubiertos por el seguro de la paciente; sin embargo, de acuerdo a la historia natural de enfermedad, el cuadro clínico, los antecedentes, el estudio de imágenes y los elevados requerimientos de insulina, a pesar del corto tiempo de diagnóstico junto al difícil manejo de la glicemia, nos llevaron a concluir que se trató de un caso de diabetes mellitus tipo 3 c secundaria a una pancreatitis crónica de etiología alcohólica.

\section{CONCLUSIONES}

La diabetes mellitus tipo 3c es aún poco diagnosticada. Su origen está circunscrito al daño de células beta pancreáticas y no es causada por anticuerpos específicos. Debido a la clasificación y caracterización por grupo etario es posible que un gran número de pacientes diagnosticados como diabetes tipo 2 no lo sean, por lo que debemos mantenernos alertas ante cada paciente diabético con historia de dolor abdominal mal referido, o buscar signos de malabsorción, para aumentar la posibilidad de un diagnóstico certero, que implicará un mejor tratamiento cuyos objetivos principales son prevenir o tratar la desnutrición, controlar los síntomas de la esteatorrea y minimizar la hiperglucemia inducida por las comidas y/o por estilos de vida que la favorecen (refuerzo de la pérdida de peso para los obesos, el ejercicio diario y los carbohidratos (imitados). Se recomienda indagar sobre el consumo de alcohol y fomentar la abstinencia de este y del tabaco, además de sugerir estilos de vida saludables a los pacientes.

Contribuciones de los autores CGD ha realizado la concepción y diseño del artículo, análisis e interpretación de datos, redacción y revisión crítica del artículo, aprobación de la versión final. JCL ha realizado la concepción del artículo, recolección de datos de la historia clínica, diseño de la investigación, aprobación de la versión final. EGR ha realizado el diseño del artículo, interpretación de datos, revisión crítica del manuscrito y aprobación de la versión final.

Fuentes de financiamiento: Este artículo ha sido financiado por los autores.

Conflictos de interés: Los autores declaran no tener ningún conflicto de interés.

\section{REFERENCIAS BIBLIOGRÁFICAS}

1. Pham A, Forsmark C. Chronic pancreatitis: review and update of etiology, risk factors, and management. F1000Res. 2018; 7: F1000.

2. De la Iglesia-García D, Huang W, Szatmary P, Baston-Rey I, GonzalezLopez J, Prada-Ramallal G, et al. Efficacy of pancreatic enzyme replacement therapy in chronic pancreatitis: systematic review and meta-analysis. Gut. 2017; 66(8): 1354-5.

3. Czul F, Coronel E, Donet JA. Una actualización de pancreatitis crónica: 
artículo de revisión. Rev Gastroenterol Perú. 2017; 37(2): 146-55.

4. Manohar M, Verma AK, Venkateshaiah SU, Sanders NL, Mishra A. Pathogenic mechanisms of pancreatitis. World J Gastrointest Pharmacol Ther. 2017; 8(1): 10-25.

5. Lundberg R, Beilman GJ, Dunn TB, Pruett TL, Freeman ML, Ptacek PE, et al. Early alterations in glycemic control and pancreatic endocrine function in non-diabetic patients with chronic pancreatitis. Pancreas. 2016; 45(4): 565-71.

6. Woodmansey C, McGovern AP, McCullough KA, Whyte MB, Munro NM, Correa AC, et al. Incidence, demographics, and clinical characteristics of diabetes of the exocrine pancreas (type3c): a retrospective cohort study. Diabetes Care. 2017; 40(11): 1486-93.

7. Ewald N, Hardt PD. Diagnosis and treatment of diabetes mellitus in chronic pancreatitis. World J Gastroenterol. 2013; 19(42): 7276-81.

8. Andersen DK, KorC M, Petersen GM, Eibl G, Li D, Rickels MR, et al. Diabetes, pancreatogenic diabetes, and pancreatic cancer. Diabetes. 2017; 66(5): 1103-10.

9. Duggan SN, Conlon KC. Pancreatogenic type $3 \mathrm{C}$ diabetes: underestimated, underappreciated and poorly managed. Pract Gastroenterol. 2017; 163: 14-23.

10. Petrov MS, Yadav D. Global epidemiology and holistic prevention of pancreatitis. Nat Rev Gastroenterol Hepatol. 2019; 16(3): 175-84.

11. Huffman JL, Obideen K, Wehbi M, Annand BS, Patel T, Talavera F, et al. Chronic pancreatitis. Medscape. 2019.

12. Drewes AM, Kempeneers MA, Andersen DK, Arendt-Nielsen L, Besselink MG, Boermeester MA, et al. Controversies on the endoscopic and surgical management of pain in patients with chronic pancreatitis: pros and cons!. Gut. 2019; 68(8): 1343-51.

13. Fujii M, Ohno Y, Yamada M, Kamada Y, Miyoshi E. Impact of fatty pancreas and lifestyle on the development of subclinical chronic pancreatitis in healthy people undergoing a medical checkup. Environ Health Prev. 2019; 24: 10.

14. Singhvi A, Yadav D. Myths and realities about alcohol and smoking in chronic pancreatitis. Curr Opin Gastroenterol. 2018; 34(5): 355-61.

15. Hao L, Wang L-S, Liu Y, Wang T, Guo H-L, Pan J, et al. The different course of alcoholic and idiopathic chronic pancreatitis: a long-term study of 2,037 patients. PLOS One. 2018; 13(6): e0198365.

16. Manikkavasakar S, AlObaidy M, Busireddy KK, Ramalho M, Nilmini V, Alagiyawanna $M$, et al. Magnetic resonance imaging of pancreatitis: an update. World J Gastroenterol. 2014; 20(40): 14760-77.

17. Rickels MR, Bellin M, Toledo FGS, Robertson RP, Andersen DK, Chari ST, et al. Detection, evaluation and treatment of diabetes mellitus in chronic pancreatitis: recommendations from PancreasFest 2012. Pancreatology. 2013; 13(4): 336-42.

\section{Correspondencia:}

Cintya Marisol Guibar Deza

Dirección: Mz C Lote 4 Urbanización Los Girasoles de San

Isidro II etapa. Trujillo, Perú.

Teléfono: 999393187

Correo electrónico: marisol_9287@hotmail.com

Recibido: 08 de enero de 2020

Evaluado: 03 de julio de 2020

Aprobado: 17 de agosto de 2020

(c) La revista. Publicado por Universidad de San Martín de Porres, Perú. (c) BY Licencia de Creative Commons Artículo en acceso abierto bajo términos de Licencia Creative Commons Atribución 4.0 Internacional. (http://creativecommons.org/licenses/by/4.0/)

\section{ORCID iDs}

Cintya Marisol Guibar Deza C https: / / orcid.org/0000-0001-9936-9227

José Guillermo Cabanillas Lopez 다ttps://orcid.org/0000-0001-9448-1295

Evelyn del Socorro Goicochea Ríos i https: / / orcid.org/0000-0001-9994-9184 